\title{
Vibrational spectra of small silicon monoxide cluster cations measured by infrared multiple photon dissociation spectroscopy $\dagger$
}

\author{
Etienne Garand, ${ }^{a}$ Daniel Goebbert ${ }^{b}$ Gabriele Santambrogio, ${ }^{c}$ Ewald Janssens, ${ }^{d}$ \\ Peter Lievens, ${ }^{d}$ Gerard Meijer, $^{b}$ Daniel M. Neumark ${ }^{* a e}$ and Knut R. Asmis ${ }^{* b}$
}

\author{
Received 29th October 2007, Accepted 2nd January 2008 \\ First published as an Advance Article on the web 30th January 2008 \\ DOI: $10.1039 / b 716627 c$
}

The first gas-phase infrared spectra of silicon monoxide cations $(\mathrm{SiO})_{n}{ }^{+}, n=3-5$, using multiple photon dissociation in the $550-1250 \mathrm{~cm}^{-1}$ frequency range, are reported. All clusters studied here fragment via loss of a neutral $\mathrm{SiO}$ unit. The experimental spectra are compared to simulated linear absorption spectra from calculated low energy isomers for each cluster. This analysis indicates that a "ring" isomer is the primary contributor to the $(\mathrm{SiO})_{3}{ }^{+}$spectrum, that the $(\mathrm{SiO})_{4}{ }^{+}$spectrum results from two close-lying bicyclic ring isomers, and that the $(\mathrm{SiO})_{5}{ }^{+}$ spectrum is from a bicyclic ring with a central, fourfold-coordinated $\mathrm{Si}$ atom. Experiment and theory indicate that the energies and energetic orderings of $(\mathrm{SiO})_{n}{ }^{+}$isomers differ from those for neutral $(\mathrm{SiO})_{n}$ clusters.

\section{Introduction}

Silicon-oxide nanoparticles are important constituents of the interstellar medium, since they act as catalysts for chemical reactions $^{1}$ and nucleation centers for planetesimal formation. ${ }^{2}$ Silicon particles or silicon-oxide clusters containing $\mathrm{Si}-\mathrm{Si}$ bonds are also candidates for the source of the intense extended red emission (500-800 $\mathrm{cm}^{-1}$ ) and UV-Vis extinction observed in the interstellar medium. ${ }^{3,4}$ Because $\mathrm{SiO}$ is the most abundant oxygen-bearing species in interstellar clouds, it has been proposed that formation of small silicon monoxide clusters, $(\mathrm{SiO})_{n}$, is the first step toward the formation of silicon-containing structures. ${ }^{5,6}$ However, the subsequent steps toward silicon $(\mathrm{Si})_{n}$ and silicates $\left(\mathrm{SiO}_{2}\right)_{n}$ are not well understood. Recently, silicon monoxide clusters have drawn attention in the field of nanotechnology as they play a crucial role in the growth of silicon nanowires. ${ }^{7-9}$ High yields of silicon nanowires surrounded by $\mathrm{SiO}_{2}$ sheaths were obtained from evaporation or laser vaporization of mixed $\mathrm{Si} / \mathrm{SiO}_{2}$ powder and of pure $\mathrm{SiO}$. The highest yield was obtained with targets containing equal amount of silicon and oxygen, highlighting the importance of small $(\mathrm{SiO})_{n}$ clusters in the early steps of the process. ${ }^{7}$ Several theoretical studies on $(\mathrm{SiO})_{n}$ have shown that for $n \geq 5$, formation of isomers containing a $\mathrm{Si}$ core (i.e. at least one $\mathrm{Si}-\mathrm{Si}$ bond) surrounded by a $\mathrm{SiO}_{2}$ layer is energeti-

\footnotetext{
${ }^{a}$ Department of Chemistry, University of California, Berkeley,

California94720,USA.E-mail: dneumark@berkeley.edu

${ }^{b}$ Fritz-Haber-Institut der Max-Planck-Gesellschaft, Faradayweg 4-6, D-14195 Berlin, Germany.E-mail: asmis@fhi-berlin.mpg.de

${ }^{c}$ Institut für Experimentalphysik, Freie Universität Berlin, Arnimallee 14, D-14195 Berlin, Germany

${ }^{d}$ Laboratory of Solid State Physics and Magnetism \& INPACInstitute for Nanoscale Physics and Chemistry, Katholieke Universiteit Leuven, Celestijnenlaan 200 D, B-3001 Leuven, Belgium

${ }^{e}$ Chemical Sciences Division, Lawrence Berkeley National

Laboratory, Berkeley, California 94720, USA

$\dagger$ Electronic supplementary information (ESI) available: Animations of selected vibrational modes of $(\mathrm{SiO})_{n}{ }^{+}$clusters. See DOI: $10.1039 /$ b716627c
}

cally favored. ${ }^{10-12}$ Such segregation of silicon and oxygen in $(\mathrm{SiO})_{n}$ and subsequent fragmentation ${ }^{10,13}$ of these clusters could therefore account for the formation of both silicates and silicon structures.

In this paper, we report the first gas-phase infrared (IR) spectra of silicon monoxide cluster cations, $(\mathrm{SiO})_{n}{ }^{+}$with $n=$ 3-5, using multiple photon dissociation spectroscopy. Although silicon monoxide clusters have been the subject of several theoretical studies, ${ }^{10-16}$ only limited experimental information is available. Neutral $(\mathrm{SiO})_{n}$ clusters with $n \leq 3$ have been studied with IR and Raman spectroscopy in rare-gas matrices by Ogden and co-workers. ${ }^{17-19}$ Analysis of these spectra indicated a planar, $D_{3 \mathrm{~h}}$ ring structure for $(\mathrm{SiO})_{3}$, a result borne out by the more recent calculations. Photoelectron spectra of the anion clusters $(\mathrm{SiO})_{n}{ }^{-}, n=3-5$ measured by Wang et al. ${ }^{20}$ showed electronic bands that were interpreted in terms of ring structures for all three anion clusters as well as for the neutrals created by photodetachment. The experiments and supporting theory reported here, however, indicate more complex structures for the cations, particularly for the $n=4$ and 5 clusters, where the lowest energy isomers appear to be bicyclic rings.

\section{Experimental and theoretical methods}

Experiments were performed on a new tandem mass spectrometer-ion trap system using radiation from the broadly tunable Free Electron Laser for Infrared eXperiments (FELIX). ${ }^{21}$ The new instrument will be described in detail elsewhere. Its principle of operation is similar to the instrument ${ }^{22}$ that we used previously at FELIX. Silicon monoxide cations, $(\mathrm{SiO})_{n}{ }^{+}$, were produced by laser vaporization of a pure silicon target in the throat of a $0.1 \% \mathrm{O}_{2}-\mathrm{He}$ gas supersonic expansion at 10 $\mathrm{Hz}$. The ion beam was then collimated in a He-filled ion guide and mass-selected in a quadrupole mass filter. Mass-selected clusters were guided into a cryogenically cooled radio frequency ring electrode trap filled with continuously flowing $\mathrm{He}$. 
Through many collisions with the He buffer gas, the ions were decelerated, trapped and cooled. With the present setup, internal (rotational) cluster temperatures of $\sim 70 \mathrm{~K}$ are typically reached, determined by analysis of the rovibrational band contour of the $\nu_{3}$ mode of $\mathrm{NH}_{2}{ }^{+}$. Ar. $(\mathrm{SiO})_{n}{ }^{+}$clusters were trapped and allowed to cool for at least $10 \mathrm{~ms}$ prior to irradiation with a single $5 \mu$ s FELIX macropulse. The macropulses were produced at $5 \mathrm{~Hz}$ with typical energies of $10-40 \mathrm{~mJ}$ and a bandwidth of $\sim 0.6$ full width at half maximum (FWHM) of the central wavelength. Resonant absorption of the IR radiation resulted in multiple photon dissociation (IRMPD) of the parent ion $^{23}$ and was detected by extracting the irradiated ions into a time-of-flight mass spectrometer and measuring the extent of fragmentation. IR spectra were measured in the range $8-20 \mu \mathrm{m}\left(1250-500 \mathrm{~cm}^{-1}\right)$.

To aid in the interpretation of the spectral features, electronic structure calculations at the B3LYP/cc-pVTZ level of theory were performed with the GAUSSIAN 03 package. ${ }^{24}$ For each cluster size, 10-15 different isomers were optimized. Harmonic frequencies and linear IR intensities for the lowest energy structures were then calculated.

Initial geometries were based on the extensive study of neutral structures by $\mathrm{Lu}$ et al., ${ }^{10}$ who performed both MP2 and B3LYP calculations with a 6-31G(d) basis set, and on our knowledge of chemical bonding in silicon oxide clusters. Lu et al. found that MP2 and B3LYP calculations gave nearly the same energetics for several clusters, thus justifying the use of B3LYP for optimizing the large number of structures explored in their study. We have assumed that a similar situation applies to cationic silicon oxide clusters. We used a larger basis set than $\mathrm{Lu}$ et al., but found only small differences in cation structures, energetics, and spectra using the smaller 6-311G* basis set. As an additional check, we calculated the B3LYP/cc-pVTZ harmonic frequencies and IR intensities of neutral $(\mathrm{SiO})_{3}$ and found very good agreement with the available experimental spectra. ${ }^{17,19}$

\section{Results and analysis}

Experimental IRMPD spectra of $(\mathrm{SiO})_{3}{ }^{+},(\mathrm{SiO})_{4}{ }^{+}$and $(\mathrm{SiO})_{5}{ }^{+}$are presented in the top panel of Fig. 1-3. For all the clusters studied here, the only observed fragmentation channel upon absorption of IR radiation is the loss of one $\mathrm{SiO}$ unit from the parent ion:

$$
(\mathrm{SiO})_{n}{ }^{+}+m \cdot h \nu \rightarrow \mathrm{SiO}+(\mathrm{SiO})_{n-1}{ }^{+} .
$$

The absence of other fragments suggests that the clusters were not composed of $\mathrm{O}_{2}$ molecules adsorbed onto silicon clusters. The spectra in the top panels of Fig. 1-3 represent the intensity of the $(\mathrm{SiO})_{n-1}{ }^{+}$fragment signal as a function of IR frequency. Each spectrum shows two or more bands between 900 and $1100 \mathrm{~cm}^{-1}$, and additional features between 600 and $700 \mathrm{~cm}^{-1}$. For each $(\mathrm{SiO})_{n}{ }^{+}$cluster, the three lowest energy isomers found from electronic structure calculations are presented in the three lower panels of Fig. 1-3 along with their calculated IR spectra and relative energies. Animations of selected vibrational modes are included as ESI. $\dagger$ We also calculated dissociation energies for $\mathrm{SiO}$ loss from each cluster, finding values of 2.92, 2.55 and $2.83 \mathrm{eV}$ for $(\mathrm{SiO})_{3}{ }^{+},(\mathrm{SiO})_{4}{ }^{+}$

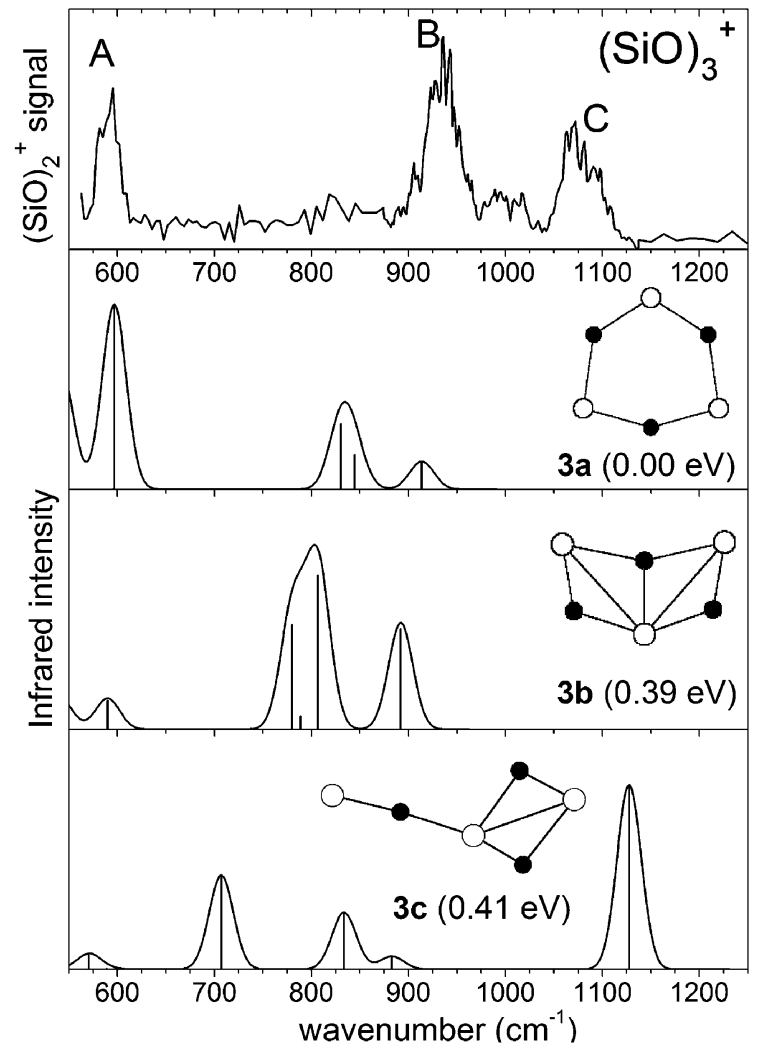

Fig. 1 Experimental and calculated spectra for the $(\mathrm{SiO})_{3}{ }^{+}$cluster. The experimental IRMPD spectrum (top panel) was recorded by measuring the intensity of the $(\mathrm{SiO})_{2}{ }^{+}$fragment. Calculated (B3LYP) structure and linear absorption spectra for the three lowest energy isomers are shown in lower panels. Black and white circles represent oxygen and silicon atoms, respectively. The calculated stick spectra were convoluted with a Gaussian line function $\left(25 \mathrm{~cm}^{-1}\right.$ FWHM).

and $(\mathrm{SiO})_{5}{ }^{+}$, respectively. Fragmentation of these clusters thus requires on the order of 20 IR photons.

The $(\mathrm{SiO})_{3}{ }^{+}$spectrum in the top panel of Fig. 1 shows three intense peaks at 590, 930 and $1080 \mathrm{~cm}^{-1}$, labeled A, B and C, respectively, and broader, weaker features between 800 and $900 \mathrm{~cm}^{-1}$ and around $1000 \mathrm{~cm}^{-1}$. Bands $\mathrm{A}$ and $\mathrm{B}$ are close to those seen in the matrix IR spectrum of neutral $\mathrm{Si}_{3} \mathrm{O}_{3}$ at 631.5 and $972.6 \mathrm{~cm}^{-1}$; these frequencies have been assigned to degenerate $\mathrm{E}^{\prime}$ stretching vibrations, for a structure comprising a six-membered ring with alternating $\mathrm{Si}-\mathrm{O}$ bonds and $D_{3 \mathrm{~h}}$ symmetry. ${ }^{17,19}$

Our calculations find that while the lowest energy structure of $(\mathrm{SiO})_{3}{ }^{+}$, structure $\mathbf{3 a}$, is a planar ring, it is less symmetric than the neutral, with $C_{2 \mathrm{v}}$ rather than $D_{3 \mathrm{~h}}$ symmetry. The calculated frequency at $600 \mathrm{~cm}^{-1}$ for a Si-O-Si stretch matches that of peak A. In the simulation, the lower symmetry for the cation splits the higher frequency degenerate vibrations into a doublet at 830 and $914 \mathrm{~cm}^{-1}$. In addition, a O-Si-O bend vibration becomes IR active at the lower symmetry, yielding an intermediate third peak at $845 \mathrm{~cm}^{-1}$. It thus appears that peak $A$ comes from structure $\mathbf{3 a}$, and while the match between peak B and C and the simulated peaks is far from perfect, the frequencies and intensities of the simulated peaks depend strongly on the extent of distortion from $D_{3 \mathrm{~h}}$ 


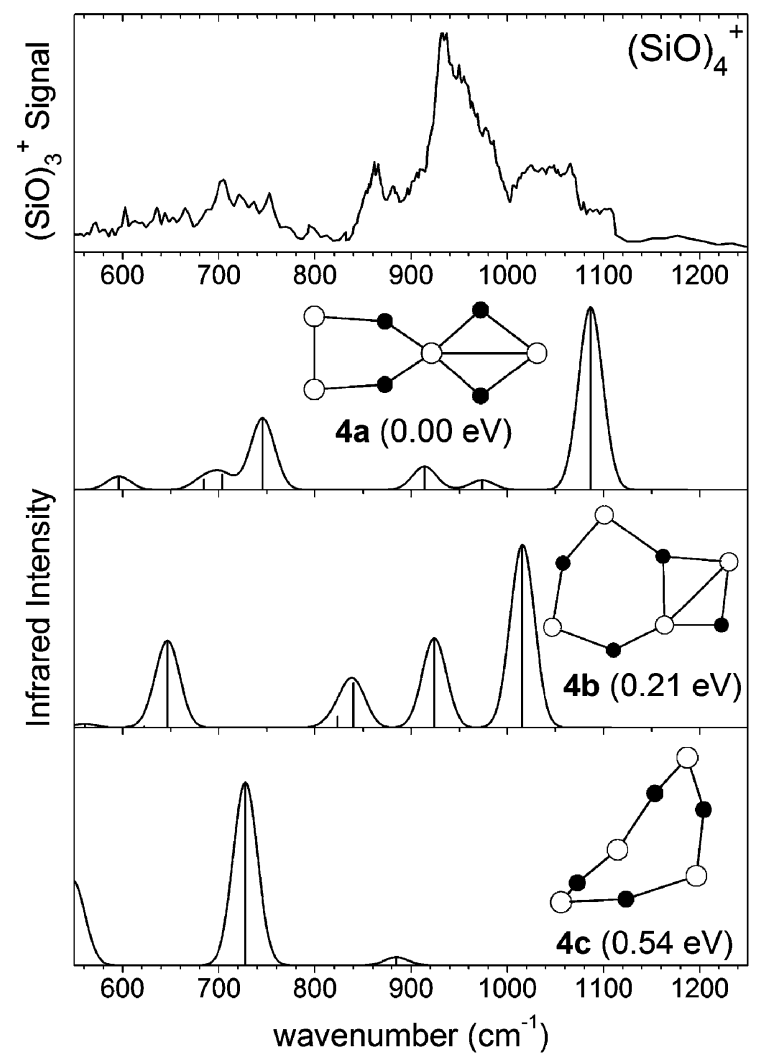

Fig. 2 Experimental and calculated spectra for the $(\mathrm{SiO})_{4}{ }^{+}$cluster. The experimental IRMPD spectrum (top panel) was recorded by measuring the intensity of the $(\mathrm{SiO})_{3}{ }^{+}$fragment. Calculated (B3LYP) structure and linear absorption spectra for the three lowest energy isomers are shown in lower panels. Black and white circles represent oxygen and silicon atoms, respectively. The calculated stick spectra were convoluted with a Gaussian line function $\left(25 \mathrm{~cm}^{-1}\right.$ FWHM $)$.

symmetry in the cation. In fact, the cation most likely experiences either dynamic or static Jahn-Teller distortion, leading to inaccurate frequencies from the type of harmonic analysis used in our simulated spectra.

Contributions from other low-lying isomers must also be considered. The lower panels in Fig. 1 show simulated spectra from the "book" isomer (3b) at $0.39 \mathrm{eV}$, and the "kite" (3c) at $0.41 \mathrm{eV}$. The most intense feature in the simulated spectrum for the kite structure at $1130 \mathrm{~cm}^{-1}$ is close to the experimental peak $\mathrm{C}$; this frequency corresponds to the antisymmetric stretch vibration of the $\mathrm{O}$ atom in the "tail" of the kite. The book structure $\mathbf{3 b}$ has a strong transition at $890 \mathrm{~cm}^{-1}$, close to the experimental peak $\mathrm{B}$. There are strong transitions in the $700-850 \mathrm{~cm}^{-1}$ region of the simulated spectrum of these isomers that are not seen in the experiment, arguing against additional contributions from these structures. However, since deviations from the linear absorption intensities are not uncommon for this type of IRMPD spectroscopy, the presence of isomer $\mathbf{3 c}$ cannot be completely ruled out. We thus attribute the $(\mathrm{SiO})_{3}{ }^{+}$spectrum to originate mainly from structure $\mathbf{3 a}$, with the caveat that a more sophisticated vibrational analysis is needed to confirm our assignment.

The $(\mathrm{SiO})_{4}{ }^{+}$experimental spectra (top panel, Fig. 2) shows five broad features centered around 700, 870, 930, 1040 and

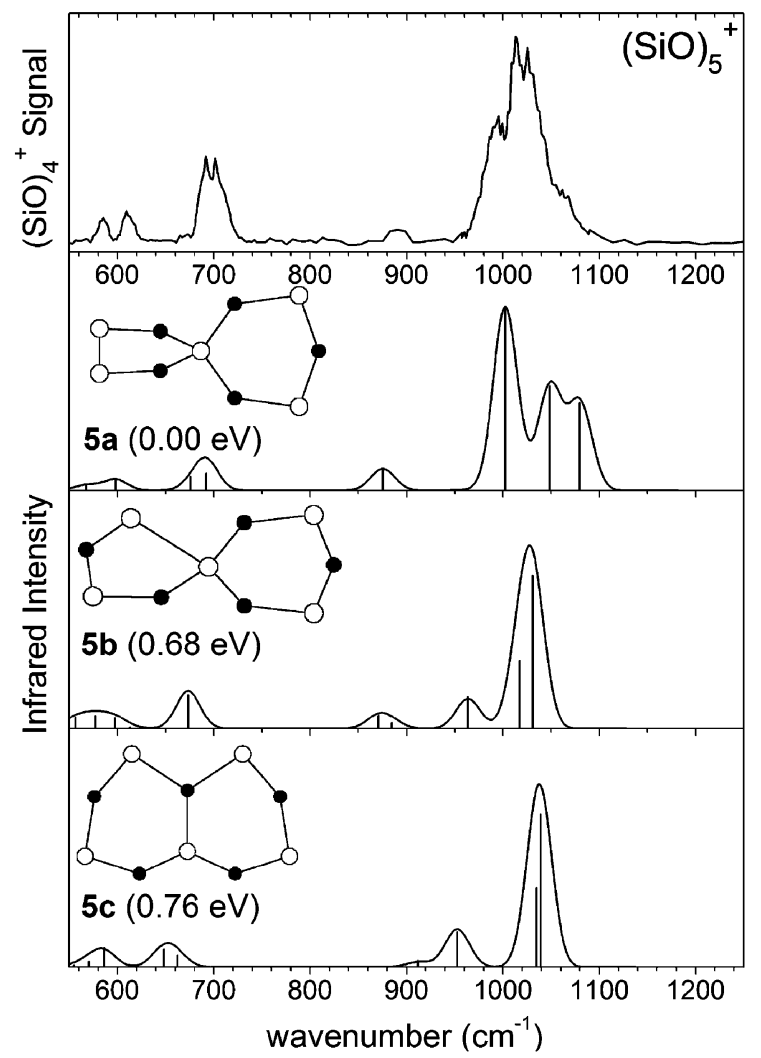

Fig. 3 Experimental and calculated spectra for the $(\mathrm{SiO})_{5}{ }^{+}$cluster. The experimental IRMPD spectrum (top panel) was recorded by measuring the intensity of the $(\mathrm{SiO})_{4}{ }^{+}$fragment. Calculated (B3LYP) structure and linear absorption spectra for the three lowest energy isomers are shown in lower panels. Black and white circles represent oxygen and silicon atoms, respectively. The calculated stick spectra were convoluted with a Gaussian line function $\left(25 \mathrm{~cm}^{-1}\right.$ FWHM $)$.

$1100 \mathrm{~cm}^{-1}$. The lowest energy $(\mathrm{SiO})_{4}{ }^{+}$isomer, structure $4 a$, found in our calculations comprises a central $\mathrm{Si}$ atom tetrahedrally coordinated to four oxygen atoms and shared between a four- and a five-membered ring. The larger ring contains a $2.98 \AA \mathrm{Si}-\mathrm{Si}$ bond, which is presumably a rather weak interaction given that the equilibrium bond length in $\mathrm{Si}_{2}$ is $2.246 \AA^{25}$ The next isomer, $4 \mathbf{b}$, lying $0.21 \mathrm{eV}$ higher in energy, has a $\mathrm{SiO}$ unit common to a six- and a four-membered ring. This structure is a bridged version of the single buckled ring isomer which was found to be $0.54 \mathrm{eV}$ higher in energy. This last isomer was found to be the minimum structure on the neutral surface, ${ }^{10-13,15,16}$ and also resembles the lowest energy structure, 3a, calculated for $(\mathrm{SiO})_{3}{ }^{+}$in Fig. 1. The calculated peak at $1090 \mathrm{~cm}^{-1}$ for structure $\mathbf{4 a}$ and the three peaks at 840 , 920 and $1010 \mathrm{~cm}^{-1}$ for structure $4 \mathbf{b}$ match peaks in the experimental spectra, suggesting that we are observing a signal from both isomers. Note that the lower frequency peaks at 750 and $650 \mathrm{~cm}^{-1}$ for $\mathbf{4 a}$ and $\mathbf{4 b}$, respectively, overlap with the broad experimental feature covering $600-750 \mathrm{~cm}^{-1}$. Contributions of multiple isomers may account for the more congested appearance of the $(\mathrm{SiO})_{4}{ }^{+}$experimental spectra compared to the two other clusters studied under the same conditions.

The electronic structure calculations show that the peak at $1090 \mathrm{~cm}^{-1}$ from structure $\mathbf{4 a}$ corresponds to antisymmetric 
stretch motion of the $\mathrm{O}$ atoms in the larger ring, while the smaller peak around $750 \mathrm{~cm}^{-1}$ is from antisymmetric stretching of the $\mathrm{O}$ atoms in the smaller ring. The higher frequency vibration is similar to the "kite tail" vibration in structure $\mathbf{3 c}$. In structure $\mathbf{4 b}$, the peaks at 1016 and $924 \mathrm{~cm}^{-1}$ correspond to the antisymmetric and symmetric stretch motion of the oxygen atoms in the larger ring, while the peaks at 840 and $647 \mathrm{~cm}^{-1}$ are associated with the doubly and triply coordinated oxygen atoms of the smaller ring.

The $(\mathrm{SiO})_{5}{ }^{+}$experimental spectrum in Fig. 3 exhibits a doublet around $600 \mathrm{~cm}^{-1}$, a more intense peak (or two peaks) at $700 \mathrm{~cm}^{-1}$, a weak feature at $890 \mathrm{~cm}^{-1}$, and three or four intense overlapped features between 970 and $1100 \mathrm{~cm}^{-1}$. The lowest energy calculated structure, 5a, has a tetrahedrallycoordinated $\mathrm{Si}$ atom common to a four-membered and a sixmembered ring, with a $2.96 \AA \mathrm{Si}-\mathrm{Si}$ bond in the smaller ring. In order of descending frequency, the three major calculated peaks for $\mathbf{5 a}$ correspond to antisymmetric $\mathrm{O}$ atom stretching in the five-membered ring, antisymmetric $\mathrm{O}$ atom stretching in the six-membered ring, and bending motion of the two $\mathrm{O}$ atoms in the larger ring adjacent to the $\mathrm{Si}$ atom. The calculated energy of 5a is substantially lower than that of structures $\mathbf{5 b}$ $(0.68 \mathrm{eV})$ and $\mathbf{5 c}(0.76 \mathrm{eV})$, which are also double-ring structures with $\mathrm{Si}$ and $\mathrm{SiO}$ cores, respectively. Structure $\mathbf{5 b}$ resembles $5 \mathbf{a}$ except that the central $\mathrm{Si}$ is bonded to a $\mathrm{Si}$ atom in the five-membered ring instead of an $\mathrm{O}$ atom. The $2.63 \AA \mathrm{Si}-\mathrm{Si}$ bond in $\mathbf{5 b}$ is also significantly shorter than in $\mathbf{5 a}$. In contrast to the situation for the smaller clusters, the $(\mathrm{SiO})_{5}{ }^{+}$experimental spectrum is matched reasonably well by that of the lowest energy calculated structure, consistent with the larger energy gap relative to the next lowest-energy structures. However the presence of the $\mathbf{5 b}$ and $\mathbf{5 c}$ isomers cannot be ruled out since their calculated spectra also agree relatively well with the experiment.

\section{Discussion}

There are several noteworthy trends in the experimental spectra. First, both experiment and theory indicate that the energetics of the considered cation isomers and even their ordering are different than their neutral counterparts. For neutral $(\mathrm{SiO})_{3}$, as discussed above, the lowest energy calculated structure by $\mathrm{Lu}$ et al. $^{10}$ is a planar ring with the next lowest, a "kite" structure, lying $1.523 \mathrm{eV}$ higher. For the cation, the kite structure $3 \mathrm{c}$ lies only $0.41 \mathrm{eV}$ above the ring, which is still found to be the lowest energy isomer, even if it is distorted in the cation. The preferred structures for $(\mathrm{SiO})_{4}{ }^{+}$ and $(\mathrm{SiO})_{5}{ }^{+}$both contain a central, tetrahedrally-coordinated silicon atom bound to four oxygen atoms and a $\mathrm{Si}-\mathrm{Si}$ bond on the periphery, and these structures account for some, if not all, of the experimental spectral features. In contrast, the lowest energy calculated structure for $(\mathrm{SiO})_{4}$ is a non-planar ring similar to $\mathbf{4 c}$, for which there is little evidence in the experimental spectrum. For neutral $(\mathrm{SiO})_{5}$, the lowest energy structure looks like $\mathbf{5 b}$, while $\mathbf{5 a}$, the apparent cation ground state, lies $0.39 \mathrm{eV}$ higher. The significance of this result is that there is a central $\mathrm{Si}-\mathrm{Si}$ bond in $\mathbf{5 b}$ but not $\mathbf{5 a}$. In the neutral clusters, this bond is predicted to evolve into a segregated $\mathrm{Si}_{n}$ core in larger clusters. ${ }^{11,12}$
Second, while we have assigned the $n=3$ and 5 to single cation isomers, two cation isomers are required to fit the $n=4$ spectrum. This result may reflect the isomer energetics, in that the calculated gap between the lowest and second lowest energy isomers is considerably smaller for $n=4$ than for the other clusters, resulting in more excited isomer population for $n=4$. Nonetheless, the apparent contribution of multiple $(\mathrm{SiO})_{4}{ }^{+}$isomers suggests that the cluster populations are well out of thermal equilibrium, given an ion temperature of $\sim 70$ $\mathrm{K}$. The structural differences between the two isomers are significant, and the clusters most likely have a complex potential energy landscape with substantial barriers to isomerization, so thermalization of the ions to room temperature in the ion guide may not be sufficient to overcome the barriers separating individual isomers. As a result, each isomer may act as a separate chemical species in the trap and cool to the bottom of its corresponding local minimum on the potential energy landscape. In an extensive study of vanadium oxide cluster ions, ${ }^{26}$ little evidence was found for the presence of multiple isomers using a similar cluster production/cooling scheme, indicating the barriers to isomerization in small $\mathrm{SiO}$ cluster cations may be significantly greater than those in vanadium oxide cluster ions.

In conclusion, we have measured the first gas-phase IR spectra of silicon monoxide cations $(\mathrm{SiO})_{n}{ }^{+}, n=3-5$, using IRMPD spectroscopy in the $550-1250 \mathrm{~cm}^{-1}$ frequency range. Comparison with the characteristic absorption regions identified by electronic structure calculation indicates that the cations have different structures than their neutral counterparts. However, exploration of the lower frequency region (300-600 $\mathrm{cm}^{-1}$ ) as well as rare-gas tagging might be necessary to pinpoint the exact structure of these species. It will also be of interest to perform experiments and electronic structure calculations on larger cluster cations to see if a $\mathrm{Si}_{n}$ central core eventually arises, since its presence would facilitate the formation of enriched silicon and silicate structures from silicon monoxide clusters. Such a mechanism could be involved in the transformation of $\mathrm{SiO}$ molecules to silicon-containing structures in the interstellar medium. ${ }^{11}$ Calculations on larger clusters may require a comprehensive search of the potential energy surface using a genetic algorithm to make sure that the global minimum is found, similar to the case of $\mathrm{Al}_{8} \mathrm{O}_{12}{ }^{+} \cdot{ }^{27}$

\section{Acknowledgements}

This work was supported by the National Science Foundation under Grant No. DMR-0139064 and by German Research Foundation (DFG) as part of the Collaborative Research Center \#546. We gratefully acknowledge the support of the "Stichting voor Fundamenteel Onderzoek der Materie" (FOM) in providing the required beam time on FELIX and highly appreciate the skillful assistance of the FELIX staff. E. G. thanks the Natural Science and Engineering Research Council of Canada for a postgraduate scholarship. D. M. N. thanks Dr Ashraf Ali for useful discussions about silicon oxide clusters.

\section{References}

1 D. A. Williams and E. Herbst, Surf. Sci., 2002, 500, 823. 
2 A. Tamanai, H. Mutschke, J. Blum and G. Meeus, Astrophys. J., 2006, 648, L147.

3 A. N. Witt, K. D. Gordon and D. G. Furton, Astrophys. J., 1998, 501, L111.

4 V. G. Zubko, T. L. Smith and A. N. Witt, Astrophys. J., 1999, 511, L57.

5 H. P. Gail and E. Sedlmayr, Astron. Astrophys., 1986, 166, 225.

6 H. P. Gail and E. Sedlmayr, Faraday Discuss., 1998, 109, 303.

7 N. Wang, Y. H. Tang, Y. F. Zhang, C. S. Lee and S. T. Lee, Phys. Rev. B, 1998, 58, 16024

8 R. Q. Zhang, Y. Lifshitz and S. T. Lee, Adv. Mater., 2003, 15, 635.

9 Y. F. Zhang, Y. H. Tang, N. Wang, D. P. Yu, C. S. Lee, I. Bello and S. T. Lee, Appl. Phys. Lett., 1998, 72, 1835.

10 W. C. Lu, C. Z. Wang, V. Nguyen, M. W. Schmidt, M. S. Gordon and K. M. Ho, J. Phys. Chem. A, 2003, 107, 6936.

11 A. C. Reber, P. A. Clayborne, J. U. Reveles, S. N. Khanna, A. W. Castleman and A. Ali, Nano Lett., 2006, 6, 1190.

12 R. Q. Zhang, M. W. Zhao and S. T. Lee, Phys. Rev. Lett., 2004, 93, 095503.

13 P. V. Avramov, I. Adamovic, K. M. Ho, C. Z. Wang, W. C. Lu and M. S. Gordon, J. Phys. Chem. A, 2005, 109, 6294.

14 R. Q. Zhang, T. S. Chu and S. T. Lee, J. Chem. Phys., 2001, 114, 5531.

15 J. R. Chelikowsky, Phys. Rev. B, 1998, 57, 3333.

16 T. S. Chu, R. Q. Zhang and H. F. Cheung, J. Phys. Chem. B, 2001, 105, 1705.

17 J. S. Anderson and J. S. Ogden, J. Chem. Phys., 1969, 51, 4189.

18 J. S. Anderson, J. S. Ogden and M. J. Ricks, Chem. Commun., 1968, 1585.

19 J. S. Ogden, Spectrochim. Acta, Part A, 1977, 33, 1059.

20 L. S. Wang, S. R. Desai, H. Wu and J. B. Nichloas, Z. Phys. D, 1997, 40, 36.
21 D. Oepts, A. F. G. van der Meer and P. W. van Amersfoort, Infrared Phys. Technol., 1995, 36, 297.

22 K. R. Asmis, M. Brümmer, C. Kaposta, G. Santambrogio, G. von Helden, G. Meijer, K. Rademann and L. Wöste, Phys. Chem. Chem. Phys., 2002, 4, 1101.

23 J. Oomens, B. G. Sartakov, G. Meijer and G. von Helden, Int. J. Mass Spectrom., 2006, 254, 1.

24 M. J. Frisch, G. W. Trucks, H. B. Schlegel, G. E. Scuseria, M. A. Robb, J. R. Cheeseman, J. A. Montgomery, Jr., T. Vreven, K. N. Kudin, J. C. Burant, J. M. Millam, S. S. Iyengar, J. Tomasi, V. Barone, B. Mennucci, M. Cossi, G. Scalmani, N. Rega, G. A. Petersson, H. Nakatsuji, M. Hada, M. Ehara, K. Toyota, R. Fukuda, J. Hasegawa, M. Ishida, T. Nakajima, Y. Honda, O. Kitao, H. Nakai, M. Klene, X. Li, J. E. Knox, H. P. Hratchian, J. B. Cross, V. Bakken, C. Adamo, J. Jaramillo, R. Gomperts, R. E. Stratmann, O. Yazyev, A. J. Austin, R. Cammi, C. Pomelli, J. Ochterski, P. Y. Ayala, K. Morokuma, G. A. Voth, P. Salvador, J. J. Dannenberg, V. G. Zakrzewski, S. Dapprich, A. D. Daniels, M. C. Strain, O. Farkas, D. K. Malick, A. D. Rabuck, K. Raghavachari, J. B. Foresman, J. V. Ortiz, Q. Cui, A. G. Baboul, S. Clifford, J. Cioslowski, B. B. Stefanov, G. Liu, A. Liashenko, P. Piskorz, I. Komaromi, R. L. Martin, D. J. Fox, T. Keith, M. A. Al-Laham, C. Y. Peng, A. Nanayakkara, M. Challacombe, P. M. W. Gill, B. G. Johnson, W. Chen, M. W. Wong, C. Gonzalez and J. A. Pople, GAUSSIAN 03 (Revision C.02), Gaussian, Inc., Wallingford, CT, 2004.

25 K. P. Huber and G. Herzberg, Molecular Spectra and Molecular Structure IV: Constants of Diatomic Molecules, Van NostrandReinhold, New York, 1977.

26 K. R. Asmis and J. Sauer, Mass Spectrom. Rev., 2007, 26, 542.

27 M. Sierka, J. Döbler, J. Sauer, G. Santambrogio, M. Brümmer, L. Wöste, E. Janssens, G. Meijer and K. R. Asmis, Angew. Chem., Int. Ed., 2007, 46, 3372. 